\title{
ON CENTRALIZERS \\ OF GENERALIZED UNIFORM SUBGROUPS OF LOCALLY COMPACT GROUPS $\left({ }^{1}\right)$
}

\author{
BY
}

\author{
KWAN-YUK LAW SIT
}

\begin{abstract}
Let $G$ be a locally compact group and $H$ a closed subgroup of $G$ such that the homogeneous space $G / H$ admits a finite invariant measure. Let $Z_{G}(H)$ be the centralizer of $H$ in $G$. It is shown that if $G$ is connected then $Z_{G}(H)$ modulo its center is compact. If $G$ is only assumed to be locally connected it is shown that the commutator subgroup of $Z_{G}(H)$ has compact closure. Consequences of these results are found for special classes of groups, such as Lie groups. An example of a totally disconnected group $G$ is given to show that the results for $Z_{G}(H)$ need not hold if $G$ is not connected or locally connected.
\end{abstract}

0 . Introduction. Let $G$ be a locally compact group and $H$ a closed subgroup of $G$ such that $G / H$ admits a finite invariant measure (we call such a subgroup a generalized uniform subgroup of $G$ ). In [1], Borel showed that the centralizer $Z_{G}(H)$ of $H$ in $G$ is equal to the center $Z(G)$ of $G$ when $G$ is a semisimple connected Lie group without compact factors. Recently, Greenleaf-Moskowitz-Rothschild [3] extended Borel's result to those connected Lie groups $G$ with the property that $Z(G)=B(G)$ where $B(G)$ is the set of bounded elements of $G$ (an element $x$ of $G$ is bounded if the conjugacy class of $x$ has compact closure). In [16] we prove a result analogous to that of Greenleaf-Moskowitz-Rothschild for linear algebraic groups.

Here we investigate compactness conditions satisfied by $Z_{G}(H)$ (see $\S 1$ for all compactness conditions on locally compact groups). S. P. Wang [19] showed that $Z_{G}(H)$ is a [Z] group and therefore an $[F D]^{-}$group (see [5]) when $G$ is a connected Lie group and $H$ is discrete. In [11], using Borel's density theorem above, D. H. Lee showed that $Z_{G}(H)$ is an $[F D]^{-}$group for

Received by the editors June 18, 1973 and, in revised form, November 8, 1973.

AMS (MOS) subject classifications (1970). Primary 22D05, 22E15, 22E40; Secondary 28A70.

Key words and phrases. Locally compact group, Lie group, periodic subset, compactness conditions, homogeneous space, invariant measure, Borel's density theorem, lattice.

( $\left.{ }^{1}\right)$ This forms part of the author's doctoral thesis at the Graduate School, City University of New York. 
connected Lie groups without assuming that $H$ is discrete. Greenleaf-MoskowitzRothschild [3] also obtained the same result as D. H. Lee by other means. Again, utilizing Borel's density theorem and a modification of a lemma of Lee we prove the following theorems.

2.1. THEOREM. Suppose that $G$ is a connected locally compact group and $H$ a generalized uniform subgroup of $G$. Then the set of periodic elements of $Z_{G}(H)$ is compact.

2.2. THEOREM. Suppose that $G$ is a locally connected locally compact group and $H$ a generalized uniform subgroup of $G$. Then the commutator subgroup of $Z_{G}(H)$ has compact closure.

From this we are able to deduce that $Z_{G}(H)$ is an $[F D]^{-}$group for a large class of locally compact groups and that in certain cases $Z_{G}(H)$ is in $[Z]$ or even abelian. Later, we give an example of a totally disconnected group $G$ such that $Z_{G}(H)$ is not an $[F D]^{-}$group. Nevertheless, when $G$ is a $p$-adic linear algebraic group, $Z_{G}(H)$ is again an $[F D]^{-}$group (see [16]).

I would like to express my gratitude and appreciation to my thesis adviser, Professor M. Moskowitz who gave much encouragement and generous help throughout the writing of this paper.

1. Notations and preliminaries. Throughout this paper, $G$ denotes a locally compact group and $H$ a closed subgroup of $G$. We first summarize some results on measure theory [7] that will be needed later. A measure $\mu$ on the left coset space $G / H$ is said to be invariant if $\mu(x E)=\mu(E)$ for all $x \in G$ and all Borel sets $E$ in $G / H$. When $H$ is invariant in $G$, then an invariant measure is a Haar measure. If $G / H$ admits a finite invariant measure, we say that $H$ is a generalized uniform subgroup of $G$ and if, in addition $H$ is discrete, then $H$ is called a lattice of $G$.

Recall that an element $g \in G$ is said to be periodic if $g$ is contained in a compact subgroup of $G$. We denote by $P(G)$ the set of all periodic elements of $G ; P(G)$ need be neither closed nor a subgroup of $G$. But, in the case where $G$ is an $[F C]^{-}$group, $P(G)$ is a closed characteristic subgroup of $G$ (see Lemma 1.5 below).

Finally, we introduce some notations and list certain classes of locally compact groups as well as several results from various places for the convenience of future reference.

For subsets $D, E$ of $G$, we denote by $\{D, E\}$ the set

$$
\left\{[d, e]=\operatorname{ded}^{-1} e^{-1} \mid d \in D, e \in E\right\},
$$

by $\langle E\rangle$ the subgroup generated by $E$ and by $E^{-}$the closure of $E$. The 
commutator subgroup of any subgroup $A$ of $G$ is denoted by $A^{\prime}$ or $[A, A]$.

(1) $[I N]=$ class of locally compact groups $G$ such that $G$ possesses a compact $I(G)$-invariant neighborhood of the identity, 1 .

(2) $[S I N]=$ class of locally compact groups $G$ such that every neighborhood of 1 in $G$ contains an $I(G)$-invariant neighborhood of 1 .

(3) $[F C]^{-}=$class of locally compact groups $G$ such that every element of $G$ is bounded, i.e. $B(G)=G$.

(4) $[F D]^{-}=$class of locally compact groups $G$ such that $G^{-}$is compact.

(5) $[F I A]^{-}=$class of locally compact groups $G$ such that $I(G)$ has compact closure in $A(G)$ where $A(G)$ is made into a topological group with the compact-open topology (for example see [6]).

(6) $[Z]=$ class of locally compact groups $G$ such that $G / Z(G)$ is compact.

1.1. Lemma (Dietzmann Lemma, see [10, p. 154]). (This result was first proved in [6].) If $E$ is a periodic subset of $G$ such that $E$ consists of bounded elements and that $E^{-}$is compact, then $\langle E\rangle^{-}$is a compact subgroup of $G$.

1.2. LemMA $[14$, p. 185]. If $G$ is discrete and is a finitely generated periodic $[F C]^{-}$group, then $G$ is finite.

1.3. Lemma $\left[6\right.$, p. 21]. A compactly generated $[F C]^{-}$group $G$ which contains a dense subset of periodic elements is compact.

1.4. Lemma [15, Theorem 3D, p. 596]. (This result is stated in [15] without proof. A proof can be found in [12].) Let $G$ be an $[F C]^{-}$group, then there exists a compact normal subgroup $K$ of $G$ such that $G$ is an extension $1 \rightarrow K \rightarrow G \rightarrow V \times D \rightarrow 1$, where $V$ is a vector group and $D \in$ $[F C]^{-}$is discrete.

1.5. Lemma (A special case of [6, Theorem 3.16, p. 21]). Let $G$ be an $[F C]^{-}$group, then $P(G)$ is a closed characteristic $[F C]^{-}$subgroup of $G$ and $G$ is an extension

$$
1 \rightarrow P(G) \rightarrow G \rightarrow V \times D \rightarrow 1,
$$

where $V$ is a vector group, $D$ a discrete torsion free abelian group, and $V \times$ $D \in[F C]^{-}$.

1.6. Lemma $[13$, p. 22]. Let $H \subset L$ be closed subgroups of $G$. If $G / H$ has finite invariant measure $\mu$, then $G / L$ and $L / H$ both admit finite invariant measures of which $\mu$ is a product. 
2. Compactness conditions of $Z_{G}(H)$. We prove Theorems (2.1) and (2.2) in a series of lemmas and propositions. In the course of the proof, we obtain some results when an $[F C]^{-}$group is an $[F D]^{-}$group (see (2.5) and (2.8)).

2.3. Lemma. Let $H$ be a generalized uniform subgroup of $G$, then $Z_{G}(H)$ is an $[F C]^{-}$group.

Proof (See [11, p. 197]). There Lee only dealt with analytic groups; but the proof is valid for any locally compact group.

2.4. CoROllary. Let $H$ be a generalized uniform subgroup of $G$. If $G$ is such that $P(G)$ is compact, then $Z_{G}(H) \in[F D]^{-}$. In particular if $G$ is aperiodic (i.e. $P(G)=\{1\}$ ), then $Z_{G}(H)$ is abelian.

Proof. Since $Z_{G}(H)$ is an $[F C]^{-}$group, it follows from (1.5) that $Z_{G}(H)^{\prime} \subset P\left(Z_{G}(H)\right)$. As $P\left(Z_{G}(H)\right)$ is contained in $P(G)$, the corollary follows easily.

2.5. Lemma. Let $G$ be an $[F C]^{-}$group and $E$ be a subset of $G$ such that for all $e \in E$, the inner automorphism $\alpha_{e}$ of $G$ induced by $e$ leaves $E$ stable $\left(\alpha_{e}(E) \subset E\right)$. Suppose that the set $\{E, E\}^{-}$is compact and that there exists a finite set $\left\{x_{1}=1, x_{2}, \cdots, x_{n}\right\}$ of distinct elements of $G$ such that $G=$ $E \cup x_{2} E \cup \cdots \cup x_{n} E$. Then $G$ is an $[F D]^{-}$group.

Proof. Set

$$
\begin{gathered}
C=\left\{x_{i} x_{j} \mid i, j=1, \cdots, n\right\}, \\
D_{1}=\{C, C\}, D_{2}=\{E, E\}, \quad D_{3}=\{C, E\}, D_{4}=\{E, C\}
\end{gathered}
$$

and

$$
D=D_{1} \cup D_{2} \cup D_{3} \cup D_{4}
$$

Then $D_{1}$ is finite and $D_{2}$ has compact closure. We write $D_{3}$ as $\bigcup_{c \in C} c\left\{e c^{-1} e^{-1} \mid e\right.$ $\in E$ \}. Since every element in $G$ has bounded $I(G)$-orbit and $C$ is a finite set, it follows that $D_{3}$ has compact closure. Similarly $D_{4}$ has compact closure and hence $D^{-}$is compact. Noticing that $D \subset G^{\prime} \subset P(G)$, we may apply (1.1) to conclude that $\langle D\rangle^{-}$is a compact subgroup of $G$. Thus (2.5) will be proved if we show that $\langle D\rangle=G^{\prime}$. For this, it suffices to show that $\{G, G\} \subset\langle D\rangle$. Indeed, take any $g, g^{\prime} \in G$, we have $g=x_{i} e$ and $g^{\prime}=x_{j} e^{\prime}$ for some $1 \leqslant i, j \leqslant n$ and $e, e^{\prime} \in E$. Hence

$$
\left[g, g^{\prime}\right]=\left[x_{i}, e\right]\left[e, e^{\prime}\right]\left[e^{\prime} e e^{\prime-1}, x_{i} x_{j}\right]\left[x_{i}, x_{j}\right]\left[x_{j} x_{i}, e^{\prime}\right]\left[e^{\prime}, x_{j}\right],
$$

where each factor in the product is an element of $D$. So $\left[g, g^{\prime}\right]$ is in $\langle D\rangle$ and the proof of $(2.5)$ is complete. 
2.6. CoRollary. Suppose that $G \in[F C]^{-}$is discrete and that $H$ is an $[F D]^{-}$subgroup of $G$ such that $G / H$ is finite. Then $G \in[F D]^{-}$.

Proof. It is obvious that $H$ is a subset of $G$ which satisfies all the hypotheses of (2.5). Hence $G^{\prime}$ is compact (finite).

2.7. LemMa. Suppose there exists a compact normal subgroup $K$ of $G$ such that $G / K$ is an $[F D]^{-}$group, then $G$ is an $[F D]^{-}$group.

Proof. Since $G^{\prime}-K / K=(G / K)^{\prime-}$ is compact, therefore $G^{\prime}-K$ and hence $G^{\prime}-$ are compact.

2.8. Proposition. Suppose that $G$ is an $[F C]^{-}$group and that $H$ is an $[F D]^{-}$subgroup of $G$ such that $G / H$ is compact. Then $G$ is an $[F D]^{-}$group.

Proof. By (1.4), there exists a compact normal subgroup $K$ of $G$ such that $G$ is an extension $1 \rightarrow K \rightarrow G \rightarrow V \times D \rightarrow 1$, where $V$ is a vector group and $D \in[F C]^{-}$is discrete. Observing that $H K / K$ is a closed subgroup of $G / K$ with $(G / K) /(H K / K)$ compact, in view of (2.7), we may assume that $G=V \times D$.

Since $V$ is an open normal subgroup of $G$, the canonical projection $\eta$ : $G \rightarrow G / V$ is both an open and a closed continuous homomorphism. Hence both $\eta(G) / \eta(H)$ and $\eta(H)^{\prime-}\left(=\eta\left(H^{\prime-}\right)\right)$ are compact (finite). Therefore by (2.6), we have $\eta(G)^{\prime-}=D^{\prime-}$ finite. Since $G^{\prime-}=D^{\prime-}$, the proof of $(2.8)$ is thus complete.

2.9. Lemma (ZASSENhaus, AUSLANDER). Let $G$ be a connected Lie group with its radical $R, \pi: G \rightarrow G / R$ the projection and $L$ a closed subgroup of $G$. If the 1-component $L_{0}$ of $L$ is solvable, then the 1-component $\left(\pi(L)^{-}\right)_{0}$ of $\pi(L)^{-}$is solvable.

2.10. COROLlary. Let $G$ be a connected Lie group whose semisimple part is compact and $L$ be a closed subgroup of $G$ such that $L_{0}$ is solvable. Then $L$ is compactly generated.

REMARK. (2.10) was obtained by D. H. Lee using (2.9) (see [11, Lemma C]) which in turn was proved by H. C. Wang [18, Theorem A] under the further assumption that $R$ is simply connected. In order to apply Wang's proof to the present case, we first generalize two technical lemmas in [18].

Let $E$ be a subset of $G$; by $L_{n}(E)$ we mean the set of commutators

$$
\left[g_{1},\left[g_{2},\left[\cdots\left[g_{n-1}, g_{n}\right] \cdots\right]\right]\right], \quad g_{i} \in E,
$$

of length $n$, and by $\lim _{n} L_{n}(E)=1$, we mean that given any neighborhood $U$ 
of 1 , there exists an integer $n_{0}$ such that $L_{n}(E) \subset U$ for all $n \geqslant n_{0}$.

2.11. LEMMA (AUSLANDER). Let $S$ be a normal subgroup of a connected Lie group $G$ and $\pi: G \rightarrow G / S$ be the projection. If $S$ is isomorphic to $T \times V$, where $T$ is a real torus and $V$ a real vector space, then there exists a neighborhood $W$ of 1 in $G / S$ such that for any compact subset $K$ of $G$ with $\pi(K) \subset W, \lim _{n} L_{n}(K)=1$.

In the case where $S$ is isomorphic to a real vector space, (2.11) is $[18$, Lemma 1, p. 210].

ProOF OF (2.11). Let $\phi: G^{\sim} \rightarrow G$ be a universal covering of $G$, $V^{\sim}=\phi^{-1}(T \times V)$; then $\phi_{\mid V} \sim: V^{\sim} \longrightarrow T \times V$ is a universal covering of $T \times V$. Let $\pi^{\sim}: G^{\sim} \rightarrow G^{\sim} / V^{\sim}$ be the projection; then we have the induced universal covering $\phi^{\sim}: G \sim / V^{\sim} \rightarrow G /(T \times V)$ which satisfies the following commutative diagram

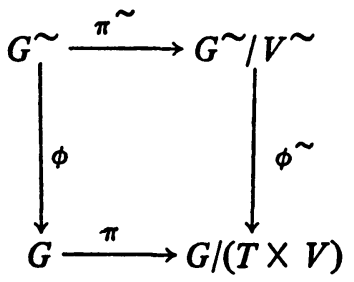

Since $V^{\sim}$ is a vector space, we have by [18, Lemma 1] a neighborhood $W^{\sim}$ of $1^{\sim}$ in $G \sim / V^{\sim}$ such that for any subset $K^{\sim}$ of $G^{\sim}$ with $\pi^{\sim}\left(K^{\sim}\right) \subset$ $W^{\sim}, \quad \lim _{n} L_{n}\left(K^{\sim}\right)=1 \sim$.

Suppose we have the following:

For any compact subset $K$ of $G$ with $\pi(K) \subset \phi^{\sim}\left(W^{\sim}\right)$, (1) there exists a compact subset $K^{\sim}$ of $G^{\sim}$ such that $\phi\left(K^{\sim}\right)$ $=K$ and $\pi\left(K^{\sim}\right) \subset W^{\sim}$.

Then $W=\phi^{\sim}\left(W^{\sim}\right)$ is a neighborhood of 1 in $G /(T \times V)$ such that for any compact subset $K$ of $G$ with $\pi(K) \subset W, \lim _{n} L_{n}(K)=1$. Indeed, let $U$ be any neighborhood of 1 in $G$, then $\phi^{-1}(U)$ is a neighborhood of $1^{\sim}$ in $G^{\sim}$. So there exists an $n_{0}$ such that $L_{n}\left(K^{\sim}\right) \subset \phi^{-1}(U)$ for all $n \geqslant n_{0}$. Hence

$$
L_{n}(K)=L_{n}\left(\phi\left(K^{\sim}\right)\right)=\phi\left(L_{n}\left(K^{\sim}\right)\right) \subset U, \quad n \geqslant n_{0} .
$$

Thus the proof of (2.11) is complete, if we establish (1).

In order to prove (1), we show first that for any $k \in K$, there exists an element $k^{\sim} \in G^{\sim}$ such that $\phi\left(k^{\sim}\right)=k$ and $\pi\left(k^{\sim}\right) \in W^{\sim}$. Indeed, let $k \in K$, then there exists a $k^{*} \in W^{\sim}$ such that $\pi(k)=\phi^{\sim}\left(k^{*}\right)$ and hence a $k_{1}^{\sim} \in G^{\sim}$ such that $\pi^{\sim}\left(k_{1}^{\sim}\right)=k^{*}$. Therefore 


$$
\pi(k)=\phi^{\sim}(\pi \sim(k \tilde{1}))=\pi(\phi(k \tilde{1})) \text { or } \phi(k \tilde{1}) k^{-1} \in T \times V .
$$

Thus there exists an $h^{\sim} \in V^{\sim}$ such that $\phi\left(h^{\sim}\right)=\phi\left(k_{1}^{\sim}\right) k^{-1}$. Let $k^{\sim}=$ $\left(h^{\sim}\right)^{-1} k_{1}^{\sim}$; then $\phi\left(k^{\sim}\right)=k$ and $\pi^{\sim}\left(k^{\sim}\right) \in W^{\sim}$.

Now let $U_{k}$ be an open neighborhood of $k^{\sim}$ in $G^{\sim}$ with $U_{k}^{-}$compact and $k^{\sim} \in U_{k} \subset U_{k}^{-} \subset\left(\pi^{\sim}\right)^{-1}\left(W^{\sim}\right)$. Then $\left\{\phi\left(U_{k}\right) \mid k \in K\right\}$ is an open covering of $K$, and hence there is a finite subcovering $\phi\left(U_{k_{1}}\right) \cup \cdots \cup \phi\left(U_{k_{n}}\right) \supset$ $K$. So the set $K^{\sim}=\phi^{-1}(K) \cap\left(U_{k_{1}}^{-} \cup \cdots \cup U_{k_{n}}^{-}\right)$has the properties specified in (1). The proof of (2.11) is thus complete.

2.12. Lemma. Let $G, S, \pi, W$ be as in (2.11), $L$ be a closed subgroup of $G$ such that $L_{0}$ is solvable. Then $L \pi^{-1}(W)$ generates a solvable group.

In the case where $S$ is isomorphic to a real vector space, (2.12) is [18, Lemma 2, p. 210]. That (2.12) follows from (2.11) is exactly the same as [18, Lemma 2] from [18, Lemma 1]; so we omit the proof here.

The proof of (2.9) is similar to that of $[18$, Theorem A] with one modification. In the induction step, we replace $G /[R, R]$ by $G /[R, R]^{-}$and the replacement is justified since $R$ is topological solvable.

We are now in the position to prove Theorems 2.1 and 2.2.

Proof of (2.1). We show first that it suffices to prove (2.1) in the case where $G$ is an analytic group. Let $K$ be a compact normal subgroup of $G$ with $G / K$ an analytic group and let $\eta: G \rightarrow G / K$ be the natural projection which is continuous, open and closed. Then $\eta(H)$ is a generalized uniform subgroup of $\eta(G)$. Hence by assumption $P\left(Z_{\eta(G)}(\eta(H))\right)$ is compact. It follows from (1.5) and (2.3) that $P\left(Z_{G}(H)\right)$ is a closed subgroup of $Z_{G}(H)$. And it is easy to see that $\eta\left(P\left(Z_{G}(H)\right)\right)$ is a closed subgroup contained in $P\left(Z_{\eta(G)}(\eta(H))\right)$. Thus $\eta\left(P\left(Z_{G}(H)\right)\right)$ and hence $P\left(Z_{G}(H)\right)$ are compact. This completes the reduction to the analytic case.

Now we assume that $G$ is an analytic group. Let $R$ be the radical of $G$ and $C$ be the maximal connected normal compact subgroup of a semisimple part of $G$. As $R C / R$ is a normal subgroup of $G / R, R C$ is a normal subgroup of $G$ and $G / R C$ is a semisimple connected Lie group without compact factors. Let $\pi: G \rightarrow G / R C$ be the natural projection. As $\pi(H)^{-}$is a closed subgroup of $\pi(G)$ such that $\pi(G) / \pi(H)^{-}\left(\cong G /(H R C)^{-}\right)$admits a finite invariant measure, so by Borel's density theorem [1], we have

$$
Z_{\pi(G)}\left(\pi(H)^{-}\right)=Z(\pi(G)) .
$$

Since $\pi\left(P\left(Z_{G}(H)\right)\right) \subset \pi\left(Z_{G}(H)\right) \subset Z_{\pi(G)}\left(\pi(H)^{-}\right)$, it follows that $\pi\left(P\left(Z_{G}(H)\right)\right)$ is an abelian discrete normal subgroup of $\pi(G)$ and hence it is finitely generated. (We note here that $\left(Z_{G}(H) R C\right) / R C=\pi\left(Z_{G}(H)\right)$ is abelian; this fact will be used in 
the proof of Corollary 2.18.) Hence by (1.2), we have $\pi\left(P\left(Z_{G}(H)\right)\right)$ finite. Now

$$
P\left(Z_{G}(H)\right) /\left(P\left(Z_{G}(H)\right) \cap R C\right) \cong \pi\left(P\left(Z_{G}(H)\right)\right)
$$

and

$$
P\left(Z_{G}(H)\right) \cap R C \subset P\left(Z_{R C}(H)\right) ;
$$

in order to prove $P\left(Z_{G}(H)\right)$ to be compact, it suffices to show that $P\left(Z_{R C}(H)\right)$ is compact.

Set $P=P\left(Z_{R C}(H)\right)$ and $P_{0}$ the connected component of 1 of $P$. Then $P_{0}$ which is a compactly generated periodic $[F C]^{-}$group is compact (see (1.3)). Let $S$ be the semisimple part of $P_{0}$ and $A(S)$ (resp. $I(S)$ ) be the topological (resp. inner) automorphism group of $S$. As $S$ is normal in $P$, we can define a homomorphism $\phi: P \rightarrow A(S)$ by $\phi(x)=\alpha_{x \mid S}, x \in P$. Since $A(S) / I(S)$ is finite and $P / \phi^{-1}(I(S)) \cong \phi(P) / I(S)$, therefore $\phi^{-1}(I(S))=Z_{P}(S) S$ has finite index in $P$. Thus $P$ will be compact if we show that $Z_{P}(S)$ is compactly generated.

Since $P_{0}=Z\left(P_{0}\right)_{0} \cdot S$, it is easy to see that $Z_{P}(S)_{0}=Z\left(P_{0}\right)_{0}$. Now $Z_{P}(S)_{0}$ $\subset Z_{P}(S) \subset R C$; therefore it follows from $(2.10)$ that $Z_{P}(S)$ is compactly generated. The proof of (2.1) is complete.

Proof of (2.2). We may assume that $H$ is normal in $G$. Since the normalizer $N_{G}(H)$ of $H$ in $G$ is a closed subgroup of $G$ containing $H$, it follows from (1.6) that $N_{G}(H) / H$ admits a finite (positive) invariant measure. Also we have $Z_{G}(H) \subset N_{G}(H)$ and $Z_{N_{G}(H)}(H)=Z_{G}(H)$.

Assuming that $H$ is normal in $G$, we have $G / H$ compact. Let $\pi: G \rightarrow$ $G / H$ be the projection, then $\left\{\pi\left(x G_{0}\right) \mid x \in G\right\}$, where $G_{0}$ denotes the 1-component of $G$, is an open covering of $G / H$. Let $x_{1}=1, x_{2}, \cdots, x_{m}$ be distinct elements of $G$ such that

$$
G / H=\bigcup_{i=1}^{m} \pi\left(x_{i} G_{0}\right) \text { and } G=\bigcup_{i=1}^{m} x_{i} G_{0} H
$$

Hence $Z_{G}(H)=\bigcup_{i=1}^{m} Z_{x_{i} G_{0} H}(H)$, and we may arrange the $x_{i}$ 's such that

$$
Z_{x_{i} G_{0} H}(H) \neq \varnothing \text { for } 1 \leqslant i \leqslant n \leqslant m
$$

and

$$
Z_{x_{i} G_{0}{ }^{H}}(H)=\varnothing \quad \text { for } n<i \leqslant m .
$$

For $2 \leqslant i \leqslant n$, pick $a_{i} \in Z_{x_{i} G_{0} H}(H)$; it is easy to see that

$$
Z_{x_{i} G_{0} H}(H)=a_{i} Z_{G_{0} H^{H}}(H) .
$$


Thus $Z_{G_{0} H}(H)$ is a subgroup of the $[F C]^{-}$group $Z_{G}(H)$ with finite index. Therefore in view of (2.8), it suffices to show that $Z_{G_{0} H}(H)$ is an $[F D]^{-}$group.

Since $G_{0} / G_{0} \cap H\left(\cong G_{0} H / H\right)$ is compact, it follows from (2.1) that $P\left(Z_{G_{0}}\left(G_{0} \cap H\right)\right)$ is compact. Thus it remains to show that

$$
Z_{G_{0} H}(H)^{\prime} \subset P\left(Z_{G_{0}}\left(G_{0} \cap H\right)\right) .
$$

Indeed let $c, c^{\prime} \in Z_{G_{0} H}(H)$ with $c=g h, g \in G_{0}$ and $h \in H$. Then

$$
\left[c, c^{\prime}\right]=g h c^{\prime} h^{-1} g^{-1} c^{\prime-1}=g c^{\prime} g^{-1} c^{\prime-1} \in G_{0} .
$$

Hence

$$
Z_{G_{0} H}(H)^{\prime} \subset G_{0} \cap Z_{G_{0} H}(H)=Z_{G_{0}}(H) \subset Z_{G_{0}}\left(G_{0} \cap H\right) .
$$

From (1.5), we see that all elements of $Z_{G_{0} H}(H)^{\prime}$ are periodic. Hence we have $Z_{G_{0} H}(H)^{\prime}$ contained in $P\left(Z_{G_{0}}\left(G_{0} \cap H\right)\right)$ and this completes the proof of (2.2).

From (2.2), we have, in particular the following theorem.

2.13. TheOREM. Let $G$ be a Lie group and $H$ a generalized uniform subgroup of $G$. Then $Z_{G}(H) \in[F D]^{-}$.

Recall that a weak pro-Lie group is a locally compact group $G$ which contains a compact normal subgroup $K$ of $G$ such that $G / K$ is a Lie group. We obtain the following corollary of (2.13).

2.14. Corollary. Let $G$ be a weak pro-Lie group and $H$ be a generalized uniform subgroup of $G$. Then $Z_{G}(H) \in[F D]^{-}$.

Proof. Let $K$ be a compact normal subgroup of $G$ such that $G / K$ is a Lie group and set $Z=Z_{G}(H)$. By (1.6), we see that $H K / K$ is a generalized uniform subgroup of $G / K$. Hence it follows from (2.13) that $Z_{G / K}(H K / K)$ $\in[F D]^{-}$. As $Z K / K \subset Z_{G / K}(H K / K)$, therefore we have $Z K / K \in[F D]^{-}$. Thus by (2.7), we have $Z K$ in $[F D]^{-}$and hence $Z \in[F D]^{-}$.

2.15. REMARK. Suppose $K$ is a compact normal subgroup of $G$ such that $Z_{G / K}(H K / K)$ is an $[F D]^{-}$group, then as shown above, we have $Z_{G}(H)$ an $[F D]^{-}$group.

2.16. CoRollary. Suppose that $G$ is solvable and connected and that $H$ is a generalized uniform subgroup of $G$. Then $Z_{G}(H)^{\prime-}$ is compact and central. 
In the special case where $G$ is analytic and $H$ is discrete this corollary was proved by S. P. Wang [20].

Proof of (2.16). Since $G$ is connected, there exists a compact normal subgroup $K$ of $G$ such that $G / K$ is an analytic group. Therefore by (2.14), we have $Z_{G}(H)^{\prime}-$ compact. Let $\pi: G \rightarrow G / K$ be the natural projection. Then $\pi(G)^{\prime-}$ is the nilradical of $\pi(G)$. Let $L$ be the unique compact subgroup of $\pi(G)^{\prime-}$. Then $L$ is central in $\pi(G)^{\prime-}$ and hence normal in $\pi(G)$. Therefore $\pi^{-1}(L)$ is normal in $G$ and, by Iwasawa [9], $\pi^{-1}(L)$ is central in G. Now $\pi\left(Z_{G}(H)^{\prime-}\right)$ is a compact subgroup contained in $L$, thus $Z_{G}(H)^{\prime-} \subset$ $\pi^{-1}(L)$ and the corollary follows.

2.17. Corollary. Let $G$ be a connected simply connected Lie group such that $G / \operatorname{rad}(G)$ has no compact factors and $H$ be a generalized uniform subgroup of $G$. Then $Z_{G}(H)$ is abelian.

This corollary has been proved by D. H. Lee [11] and in the special case where $G$ is solvable and $H$ discrete, it was proved by R. Tolimieri [17]; In fact, we can obtain the same result on $Z_{G}(H)$ by slightly weakening the conditions on $G$ (see Corollary 2.18). Corollary 2.18 was also proved by GreenleafMoskowitz-Rothschild by different methods [3].

2.18. CoRollary. Let $G$ be a connected Lie group such that $G / \operatorname{rad}(G)$ has no compact factors and let $H$ be a generalized uniform subgroup of $G$. If the center $Z(N)$ of the nilradical $N$ of $G$ is simply connected, then $Z_{G}(H)$ is abelian.

Proof. As mentioned in the proof of (2.1), we have $\left(Z_{G}(H) R C\right) / R C$ abelian where $R=\operatorname{rad}(G)$ and $C$ is the maximal connected compact normal subgroup of a semisimple part of $G$ which is trivial in this case. Therefore we have $Z_{G}(H)^{\prime}-\subset R$. Let $K$ be a maximal compact subgroup of $R$ that contains $Z_{G}(H)^{\prime-}$; then $K$ is connected and hence abelian (see [9]). Let $L$ be the identity component of the normalizer of $K$ in $R$. Since $K$ is a compact normal abelian subgroup of $L$, it follows from [9] that $K \subset Z(L)$ and in fact, $K \subset Z(L)_{0}$. Now $Z(L)_{0} \subset$ nilradical of $L=L^{\prime-} \subset R^{\prime-}=N$, and so $K$ is the unique maximal compact subgroup of $N$ and hence $K \subset Z(N)$. Since $Z(N)$ contains no compact subgroups, $K=\{1\}$ and $Z_{G}(H)$ is abelian.

2.19. CoRollaRY. Let $G$ be an [IN] group and $H$ be a generalized uniform subgroup of $G$. Then $Z_{G}(H) \in[F D]^{-}$.

Proof. As any $[I N]$ group is a weak pro-Lie group [6, Theorem 2.11], the corollary follows from (2.14) immediately. However, we shall give a different proof which is considerably more elementary. 
As in the proof of (2.2), we may assume that $H$ is normal in $G$ and that $G_{j}^{\prime} H$ is compact. Let $V$ be a compact $I(G)$-invariant neighborhood of 1 in $G$ and $U$ be an open symmetric neighborhood of 1 in $G$ such that $U^{2} \subset$ $V$. It can be seen easily that we can select $m$ distinct points $x_{1}=1, x_{2}, \cdots$, $x_{m}$ in $G$ such that

$$
G=\bigcup_{i=1}^{m} x_{i} U H \text { and } Z_{G}(H)=\bigcup_{i=1}^{n} Z_{x_{i} U H}(H)
$$

where $1 \leqslant n \leqslant m$ and

$$
Z_{x_{i} U H}(H) \neq \varnothing \quad \text { for } 1 \leqslant i \leqslant n ; \quad Z_{x_{i} U H}(H) \neq \varnothing \text { for } n<i \leqslant m .
$$

For each $1 \leqslant i \leqslant n$, pick an $a_{i} \in Z_{x_{i} U H}(H)$ with $a_{1}=1$, then it is easy to see that $x_{i} U H \subset a_{i} V H$. Thus

$$
Z_{G}(H)=\bigcup_{i=1}^{n} Z_{a_{i} V H}(H)=\bigcup_{i=1}^{n} a_{i} Z_{V H}(H) .
$$

If we show that $Z_{V H}(H)$ is $I(G)$-invariant and that $\left\{Z_{V H}(H), Z_{V H}(H)\right\}^{-}$ is compact, then we shall have $Z_{G}(H) \in[F D]^{-}$by (2.5). It is obvious that $Z_{V H}(H)$ is $I(G)$-invariant since both $V H$ and $Z_{G}(H)$ are $I(G)$-invariant and $Z_{V H}(H)=V H \cap Z_{G}(H)$. Furthermore, we have $\left\{Z_{V H}(H), Z_{V H}(H)\right\}$ contained in $V V^{-1}$. Indeed, for any $x, x^{\prime} \in Z_{V H}(H)$, let $x^{\prime}=v h$ with $v \in V$ and $h \in H$, then

$$
\left[x, x^{\prime}\right]=x v h x^{-1} h^{-1} v^{-1}=x v x^{-1} v^{-1} \in V V^{-1} .
$$

Hence $\left\{Z_{V H}(H), Z_{V H}(H)\right\}^{-}$is compact and this completes the proof of (2.19).

If we impose some conditions on the generalized uniform subgroup $H$ instead of on $G$, then there are many cases where $Z_{G}(H)$ becomes an $[F D]^{-}$ group or even a $[Z]$ group (since $[F D]^{-} \subset[Z]$, see $[5$, p. 331]) as shown in the proposition below.

2.20. Proposition. Let $G$ be a locally compact group and $H$ be a generalized uniform subgroup of $G$.

(i) If $H$ is such that $H / H_{0}$ is compact, then $Z_{G}(H) \in[F D]^{-}$.

(ii) If (a) $H$ is a Lie group such that $\operatorname{rad}\left(H_{0}\right)$ is compact, or (b) $H$ is compactly generated such that $H_{0}$ is open and compact, then $Z_{G}(H) \in$ $[F D]^{-}$.

(iii) If $H$ is an $[F D]^{-}$group, then so is $Z_{G}(H)$.

(iv) If $H$ is abelian, then $Z_{G}(H) \in[Z]$.

(v) If $I(H)$ is closed in $A(H)$, then $Z_{G}(H) \in[Z]$.

In particular, if one of the following conditions is satisfied, then $I(H)$ is closed in $\mathrm{A}(\mathrm{H})$ and $(\mathrm{v})$ follows. 
(a) $H$ is a semisimple Lie group [4, p. 337].

(b) $H$ is a connected Lie group and has a lattice [2]. (In particular, $H$ is a Q-rational simply connected nilpotent Lie group-a classical result of Malcev.)

(c) $H$ is $a .[Z]$ group.

(d) $H$ is discrete and finitely generated. (In the particular case where $G$ is an analytic group and $H$ discrete (hence finitely generated), it was proved by $S$. $P$. Wang [19] that $Z_{G}(H)$ is an [Z] group.)

Proof of (2.20). As mentioned in the proof of (2.2), we may assume that $H$ is normal in $G$ and that $G / H$ is compact.

(v) Let $\eta: G \rightarrow A(H)$ be the continuous homomorphism defined by $\eta(g)=\alpha_{g \mid H}$. Then $\eta^{-1}(I(H))=Z_{G}(H) H$ is a closed subgroup of $G$ and hence $Z_{G}(H) H / H$ is compact. Since the map $Z_{G}(H) \rightarrow Z_{G}(H) H / H$ is continuous and the compactness of $Z_{G}(H) H / H$ implies that $Z_{G}(H)$ is $\sigma$-compact, so the map is open and hence $Z_{G}(H) / Z_{G}(H) \cap H$ is topological isomorphic to $Z_{G}(H) H / H$. Now $Z_{G}(H) \cap H \subset \operatorname{center}\left(Z_{G}(H)\right)$, therefore $Z_{G}(H) /$ center $\left(Z_{G}(H)\right)$ is compact and (v) is proved.

(iv) It is easy to see that $H \subset \operatorname{center}\left(Z_{G}(H)\right) \subset Z_{G}(H)$. Since, by assumption, $G / H$ is compact, it follows that $Z_{G}(H) \in[Z]$.

(iii) Assuming that $H$ is a normal $[F D]^{-}$subgroup of $G$, we have $H^{\prime-}$ a compact normal subgroup of $G$. Therefore $H / H^{\prime-}$ is an abelian normal subgroup of $G / H^{\prime-}$ with compact quotient and it follows from (iv) that $Z_{G / H^{\prime}}\left(H / H_{-}^{\prime}\right) \in[Z]$ and in particular in $[F D]^{-}$. Thus by Remark 2.15, we have $Z_{G}(H) \in[F D]^{-}$.

(ii) (a) Here $\operatorname{rad}\left(H_{0}\right)$ is a compact normal subgroup of $G$ and $H / \operatorname{rad}\left(H_{0}\right)$ is a semisimple Lie group with compact quotient in $G / \operatorname{rad}\left(H_{0}\right)$. Since $I\left(H / \operatorname{rad}\left(H_{0}\right)\right)$ is closed in $A\left(H / \operatorname{rad}\left(H_{0}\right)\right)$ (see [7, p. 337]), it follows from (v) that $Z_{G / \operatorname{rad}\left(H_{0}\right)}\left(H / \operatorname{rad}\left(H_{0}\right)\right)$ is an $[F D]^{-}$group and hence $Z_{G}(H) \in[F D]^{-}$ by (2.15).

(b) Since $H / H_{0}$ is discrete and finitely generated, it follows that $I\left(H / H_{0}\right)$ is closed in $A\left(H / H_{0}\right)$ and as in the proof of (a) above, we have $Z_{G}(H) \in[F D]^{-}$

(i) Since both $H / H_{0}$ and $\left(G / H_{0}\right) /\left(H / H_{0}\right)$ are compact, we have $G / H_{0}$ and hence $G / G_{0}$ compact. Therefore $G$ is a weak pro-Lie group and (i) follows from (2.14).

We now give an example of a totally disconnected group $G$ with a discrete $[F C]^{-}$generalized uniform subgroup $H$ such that $Z_{G}(H)$ is not an $[F D]^{-}$ group. (See [16] for a class of totally disconnected groups such that $Z_{G}(H) \in$ $[F D]^{-}$.)

Let $H$ be a weak direct sum of countably many copies of a finite simple group, discretely topologized. It is obvious that $H \in[F C]^{-} \cap[S I N]=[F I A]^{-}$ 
(see [5, Theorem 4.1, p. 325]), that is, $I(H)^{-}$is compact. Let $G$ be the semidirect product $H \times_{\eta} I(H)^{-}$, where $\eta(\alpha)(h)=\alpha(h), h \in H$ and $\alpha \in I(H)^{-}$, with the product topology (see [11]). Then $H$ is a closed normal subgroup of $G$ with $G / H$ compact. Here $G / H$ has finite invariant measure. An element $(h, \alpha) \in$ $Z_{G}(H)$ if and only if for all $h^{\prime} \in H$,

$$
\left(h^{\prime}, \text { id }\right)=(h, \alpha)\left(h^{\prime}, \text { id }\right)(h, \alpha)^{-1}=\left(h \alpha\left(h^{\prime}\right) h^{-1}, \text { id }\right),
$$

in other words, if and only if $\alpha=\alpha_{n}-1$.

Now consider the map $\phi: H \rightarrow Z_{G}(H)$ defined by $\phi(h)=\left(h, \alpha_{h-1}\right)$. It is obvious that $\phi$ is an one-one onto continuous map such that $\phi(h k)=\phi(k) \phi(h)$, $h, k \in H$ (i.e. $\phi$ is an antihomomorphism). Since $H$ is a countable infinite discrete group, $Z_{G}(H)=\phi(H)$ is a countable union of closed subsets, namely $\bigcup_{h \in H}\left\{\left(h, \alpha_{h^{-1}}\right)\right\}$. Hence one of the sets $\phi(\{h\})=\left\{\left(h, \alpha_{h-1}\right)\right\}$ has a nonvoid interior and it follows that $Z_{G}(H)$ is discrete and $\phi$ is a homeomorphism. We claim that $Z_{G}(H)^{\prime-}$ is not compact. For if $Z_{G}(H)^{\prime-}$ were compact, then using the fact that $\phi$ is an antihomomorphism and a homeomorphism, we have $\phi\left(H^{\prime-}\right)=\phi(H)^{\prime-}=$ $Z_{G}(H)^{\prime-}$ and hence $H^{\prime-}$ would be compact. But $H=H^{\prime-}$ is infinite (not compact). So the claim is proved.

\section{REFERENCES}

1. A Borel, Density properties for certain subgroups of semi-simple groups without compact components, Ann. of Math. (2) 72 (1960), 179-188. MR 23 \#A964.

2. H. Garland and M. Goto, Lattices and the adjoint group of a Lie group, Trans. Amer. Math. Soc. 124 (1966), 450-460. MR 33 \#7459.

3. F. Greenleaf, M. Moskowitz and L. P. Rothschild, Compactness of certain homogeneous spaces of finite volume, Amer. J. Math. (to appear).

4. S. Grosser, O. Loos and M. Moskowitz, Über Automorphismengruppen lokal-kompakter Gruppen und Derivationen von Lie-Gruppen, Math. Z. 114 (1970), 321-339.

MR 41 \#8575.

5. S. Grosser and M. Moskowitz, On central topological groups, Trans. Amer. Math. Soc. 127 (1967), 317-340. MR 35 \#292.

6. - Compactness conditions in topological groups, J. Reine Angew. Math. 246 (1971), 1-40. MR 44 \#1766.

7. E. Hewitt and K. Ross, Abstract harmonic analysis. Vol. I: Structure of topological groups. Integration theory, group representations, Die Grundlehren der math. Wissenschaften, Band 115, Academic Press, New York; Springer-Verlag, Berlin, 1963. MR 28 \#158.

8. G. Hochschild, The structure of Lie groups, Holden-Day, San Francisco, 1965. MR 34 \#7696.

9. K. Iwasawa, On some types of topological groups, Ann. of Math. (2) 50 (1949), 507-558. MR 10, 679.

10. A. G. Kuros, Theory of groups, 2nd ed., GITTL, Moscow, 1953; English transl., Chelsea, New York, 1955. MR 15, 501; 17, 124. 
11. D. H. Lee, On the centralizer of a subgroup of a Lie group, Proc. Amer. Math. Soc. 30 (1971), 195-198. MR 44 \#367.

12. J. R. Liukkonen, Dual spaces of groups with precompact conjugacy classes, Trans. Amer. Math. Soc. 180 (1973), 85-108.

13. G. D. Mostow, Homogeneous spaces with finite invariant measure, Ann. of Math. (2) 75 (1962), 17-37. MR 26 \#2546.

14. B. H. Neumann, Groups with finite classes of conjugacy elements, Proc. London Math. Soc. (3) 1 (1951), 178-187. MR 13, 316.

15. L. C. Robertson, $A$ note on the structure of Moore groups, Bull. Amer. Math. Soc. 75 (1969), 594-599. MR 39 \#7027.

16. K. Y. L. Sit, On bounded elements and centralizers of generalized uniform subgroups of locally compact groups, Doctoral thesis, The City Univ. of New York, New York, 1973.

17. R. Tolimieri, On the Selberg conditions for subgroups of solvable Lie groups, Bull. Amer. Math. Soc. 77 (1971)) 584-586. MR 45 \#448.

18. H. C. Wang, On the deformation of a lattice in a Lie group, Amer. J. Math. 85 (1963), 189-212. MR 27 \# 2582.

19. S. P. Wang, On the centralizer of a lattice, Proc. Amer. Math. Soc. 21 (1969), 21-23. MR 38 \#5989.

20. - On S-subgroups of solvable Lie groups, Amer. J. Math. 92 (1970), 389-397. MR 41 \#8581.

DEPARTMENT OF MATHEMATICS, GRADUATE CENTER, CITY UNIVERSITY OF NEW YORK, NEW YORK, NEW YORK 10036

Current address: Department of Mathematics, York College, City University of New York, New York, New York 11432 